University of Wollongong

Research Online

Faculty of Engineering and Information

Faculty of Engineering and Information

Sciences - Papers: Part B

Sciences

2019

\title{
Measurement and analysis of the radial motion error of aerostatic ultra- precision spindle
}

\author{
Hailong Cui \\ China Academy of Engineering Physics, hcui@uow.edu.au \\ Dajiang Lei \\ China Academy of Engineering Physics \\ Xinjiang Zhang \\ China Academy of Engineering Physics \\ He Lan \\ China Academy of Engineering Physics \\ Zhengyi Jiang \\ University of Wollongong, jiang@uow.edu.au
}

See next page for additional authors

Follow this and additional works at: https://ro.uow.edu.au/eispapers1

Part of the Engineering Commons, and the Science and Technology Studies Commons

Research Online is the open access institutional repository for the University of Wollongong. For further information contact the UOW Library: research-pubs@uow.edu.au 


\title{
Measurement and analysis of the radial motion error of aerostatic ultra-precision spindle
}

\author{
Abstract \\ The measurement of the rotation error of an aerostatic ultra-precision spindle is critically important to \\ evaluate and hence ensure the precision of machine tools. The Donaldson reversal method, which was \\ taken as the efficient method for error separation theoretically, has been widely used to separate shape \\ errors of standard artifact. However, the accuracy analysis of the Donaldson reversal method has not \\ been fully studied and understood. In this study, a nanometer system for measuring the radial rotation \\ error of aerostatic ultra-precision spindle was constructed based on the Donaldson reversal method. The \\ comparative experiments were carried out to investigate the effects of the motor drive, and an angle \\ correction algorithm was proposed to alleviate the effect of angle deviation. The method of harmonic \\ analysis was applied to investigate the effect of artifact eccentricity, and the relationship between the \\ axial motion and measuring error was also studied. The measuring accuracy can be improved by reducing \\ the cogging torque of motor, the angle deviation, artifact eccentricity and spindle axial motion. \\ Experimental results showed that the measurement uncertainty of both the spindle rotation error and \\ artifact form error can be controlled in nanometer level. Besides, the separated value of the artifact form \\ error was very close to the nominal roundness, which verifies the accuracy of the measurement system \\ and the validity of the error separation method.

\section{Disciplines} \\ Engineering | Science and Technology Studies

\section{Publication Details} \\ Cui, H., Lei, D., Zhang, X., Lan, H., Jiang, Z. \& Kong, L. (2019). Measurement and analysis of the radial \\ motion error of aerostatic ultra-precision spindle. Measurement: Journal of the International \\ Measurement Confederation, 137 624-635.
}

\section{Authors}

Hailong Cui, Dajiang Lei, Xinjiang Zhang, He Lan, Zhengyi Jiang, and Lingbao Kong 


\title{
Measurement and analysis of the radial motion error of aerostatic ultra-precision spindle
}

\begin{abstract}
The measurement of the rotation error of an aerostatic ultra-precision spindle is critically important to evaluate and hence ensure the precision of machine tools. The Donaldson reversal method, which was taken as the efficient method for error separation theoretically, has been widely used to separate shape errors of standard artifact. However, the accuracy analysis of the Donaldson reversal method has not been fully studied and understood. In this study, a nanometer system for measuring the radial rotation error of aerostatic ultra-precision spindle was constructed based on the Donaldson reversal method. The comparative experiments were carried out to investigate the effects of the motor drive, and an angle correction algorithm was proposed to alleviate the effect of angle deviation. The method of harmonic analysis was applied to investigate the effect of artifact eccentricity, and the relationship between the axial motion and measuring error was also studied. The measuring accuracy can be improved by reducing the cogging torque of motor, the angle deviation, artifact eccentricity and spindle axial motion. Experimental results showed that the measurement uncertainty of both the spindle rotation error and artifact form error can be controlled in nanometer level. Besides, the separated value of the artifact form error was very close to the nominal roundness, which verifies the accuracy of the measurement system and the validity of the error separation method.
\end{abstract}

Keywords: Donaldson reversal method; Angle deviation; Harmonic analysis; Rotation error; Aerostatic ultra-precision spindle.

\section{Introduction}

Aerostatic ultra-precision spindles, which are nearly frictionless and require low driving power and possess high accuracy of movement, have been widely used in ultra- 
precision machine tools and measurement equipment [1-2]. The machining and measurement accuracy of these instruments was significantly influenced by the error motions of the aerostatic ultra-precision spindle [3-5]. In recent years, many reports have focused on the method to reduce the rotation error of aerostatic ultra-precision spindle to nanometer level [6]. Therefore, the rotation error measurement of aerostatic ultra-precision spindle is an essential method to evaluate and improve the precision of machine tools [7]. The aerostatic ultra-precision spindle error motions measurement normally includes radial error, axial error and angular error. Compared with the axial error and angular error, radial rotation error is much more difficult to be accurately measured, since the radial rotation error is affected by many factors, such as position error of sensors and artifact, eccentricity of artifact, roundness of artifact, etc. The instruments hardware manufacturing and installation precision, sampling accuracy, method for separating the eccentricity error and artifact form error from the measurement results should all be considered to realize the nanometer level measurement for spindle rotation error.

Throughout the past few years, many different techniques were proposed to reduce the uncertainty of radial rotation error measurements, and the most common error separation methods are multistep method, multipoint method and reversal method [8]. Linxiang [9] presented the measurement accuracy of multistep method and appropriate number of sampling points by theoretical analysis and experimental verification, and pointed out that the spindle system error and roundness error of the workpiece cannot be completely separated by the multistep method. Buajarern et al. [10] investigated the effect of step number on the roundness error separation using multistep method, indicated that the number of step played an important role in the accuracy level of measurement, and at least 10 steps needed to satisfy the nanometer level. Haitjema [11] proposed a combination step method to enhance error separation and reduce amount of measurements, presented that the standard 12-step can be reduced to 7-step, and the uncertainty can be achieved in $4 \mathrm{~nm}$.

An alternate method to multistep is the multipoint method, which only requires one step to complete measurements [12-13]. The most common multipoint method is the 
three-point method [14]. Because there is no need to reinstall the sensors and artifact, the multipoint method is widely applied in on-machine measurement [15-16]. However, the three-point method has the critical problem of harmonic suppression. In order overcome this drawback of three-point method, Gao et al. [17] proposed a mixed method to capture the high-frequency components. Tu et al. [18] presented an exact geometric model and error analysis for the conventional three-point method, suggested that the reference artifact accuracy should be at least ten times better than that of the axis motion, and the mounting error of the probes should be less than one fiftieth of the artifact size. Cappa et al. [19] optimized the probes angles to alleviate the problem of harmonic suppression, and the effects of unequal sensor sensitivities were eliminate by a new method, which only required a single sensor. The uncertainty of Cappa's method showed the measurement uncertainty of $0.455 \mathrm{~nm}$. Recently, Shi et al. [20] proposed a hybrid three-method to investigate the harmonic measurement errors, and demonstrated that the conventional three-probe measurements need to be performed for optimizing individual Fourier coefficients.

Compared with the multipoint method, the reversal method can completely separate the artifact form error from the spindle motion error [21]. However, the conventional reversal method requires the $180^{\circ}$ rotation of sensor and artifact, which inevitably results in the position errors. To eliminate the effect of position errors, Grejda et al. [22] proposed an improvement method, which used a high precision indexing table and a reversal chuck to relocate the sensor and artifact. The technique proposed by Grejda can achieve sub-nanometer repeatability and nanometer reproducibility. Marsh et al. [23] conducted a comparison of reversal and multipoint error separation, and showed that both the spindle error and artifact form error can be reliably and repeatedly measured in sub-nanometer by both reversal and multipoint technique. Another nanometer-level comparison of Donaldson method, multipoint and multistep method was also presented by Marsh et al. [24], the measurement results demonstrated subnanometer level repeatability in the measurement of the combined spindle error motion and artifact form error. Although these were believed to be the most repeatable results, the multipoint method still exist the problem of inability to separate specific harmonics 
of the spindle error motion from the artifact form error.

It is well known that the reversal method can realize the completely separation in theory if only measurement errors can be controlled in a reasonable range. Cappa et al. [19] showed the error sources analysis on the Donaldson reversal method, Grejda reversal method and multipoint method, the effects of position errors of artifact and sensor, tilt and misalignment error of sensor, artifact eccentricity on measurement accuracy were quantitatively calculated, and pointed out that the largest source of uncertainty for Donaldson reversal method was eccentricity. Marsh et al. [24] also pointed out the spindle drive can have a significant influence on the synchronous component of the measured data. Some other factors, which are rarely mentioned in the existed literatures, also have influences on the measurement results, such as spindle axial motion error, angle accuracy of sampling. Up to now, these factors have not been systematically investigated.

Therefore, this paper concentrates on the influences of spindle motor drive, angle accuracy of sampling, artifact eccentricity, and spindle axial motion error on the radial motion accuracy. First, a nanometer system for measuring the rotation error of aerostatic ultra-precision spindle was constructed based on the Donaldson reversal method. Second, the comparative experiments were carried out to investigate the effects of the motor drive on the running accuracy, an angle correction algorithm was proposed to alleviate the effect of angle deviation on the measuring accuracy, the method of harmonic analysis was applied to investigate the effect of artifact eccentricity on the measurement results, and the relationship between the axial motion and measuring error was also studied. Finally, the experimental validation for repeatability of the measuring results was conducted, and the uncertainty analysis was also presented.

\section{Principle and measuring system}

\subsection{Principle of Donaldson reversal method}

The principle of Donaldson reversal method is shown in Fig.1. The artifact was installed at the end of aerostatic ultra-precision spindle. Both the artifact and sensor were required to rotate $180^{\circ}$ precisely between two measurements. The distance 
between the artifact and the sensor was recorded by the displacement sensor. Because different sensor sensitivities will result in the measurement error, only single displacement sensor is applied in the Donaldson reversal method.

The measurement data included the form error of the artifact and the rotation error of aerostatic spindle. The relationship of two errors can be expressed by the following equations:

$$
\begin{array}{r}
T\left(\theta_{i}\right)=S\left(\theta_{i}\right)+R\left(\theta_{i}\right) \\
T^{\prime}\left(\theta_{i}\right)=-S\left(\theta_{i}\right)+R\left(\theta_{i}\right)
\end{array}
$$

where $T\left(\theta_{i}\right)$ is the measurement data of before reverse, $T^{\prime}\left(\theta_{i}\right)$ is the measurement data of after reverse, $S\left(\theta_{i}\right)$ is the roundness form of the standard ball, $R\left(\theta_{i}\right)$ is the radial rotation error of aerostatic ultra-precision spindle. According to Eqs.(1) and (2), the rotation error of aerostatic spindle was separated from the artifact form error:

$$
\begin{aligned}
& R\left(\theta_{i}\right)=\frac{T\left(\theta_{i}\right)+T^{\prime}\left(\theta_{i}\right)}{2} \\
& S\left(\theta_{i}\right)=\frac{T\left(\theta_{i}\right)-T^{\prime}\left(\theta_{i}\right)}{2}
\end{aligned}
$$

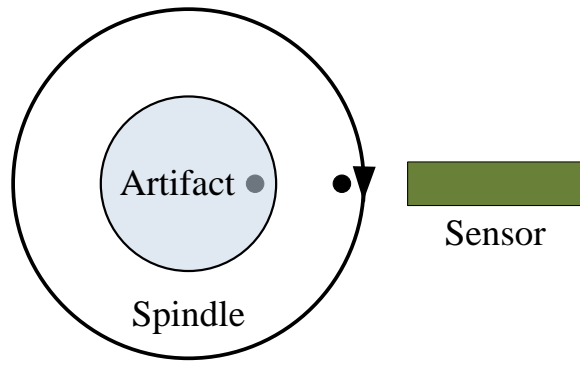

Before reverse

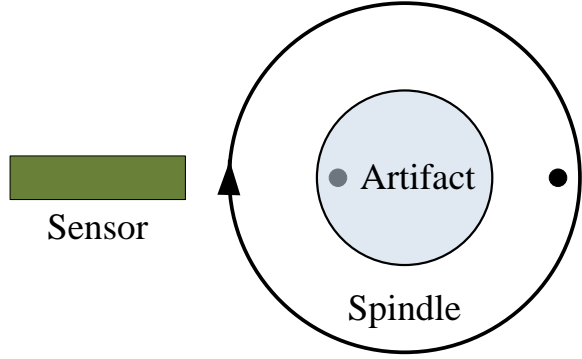

After reverse

Fig.1 Principle of Donaldson reversal method

\subsection{Measuring system}

A nanometer system for measuring the rotation error of aerostatic ultra-precision spindle is shown Fig.2. The measuring system was mainly comprised by the following parts: control system and results display, marble base and vibration isolating system, protective shield and measuring platform. The movement of aerostatic spindle and the collection of measuring data were dominated by the control system. The vibration 
isolating system and marble base were applied to reduce the influence of surrounding vibration on the measurement results. The effect of environmental noise and airflow can be restricted by the protective shield.

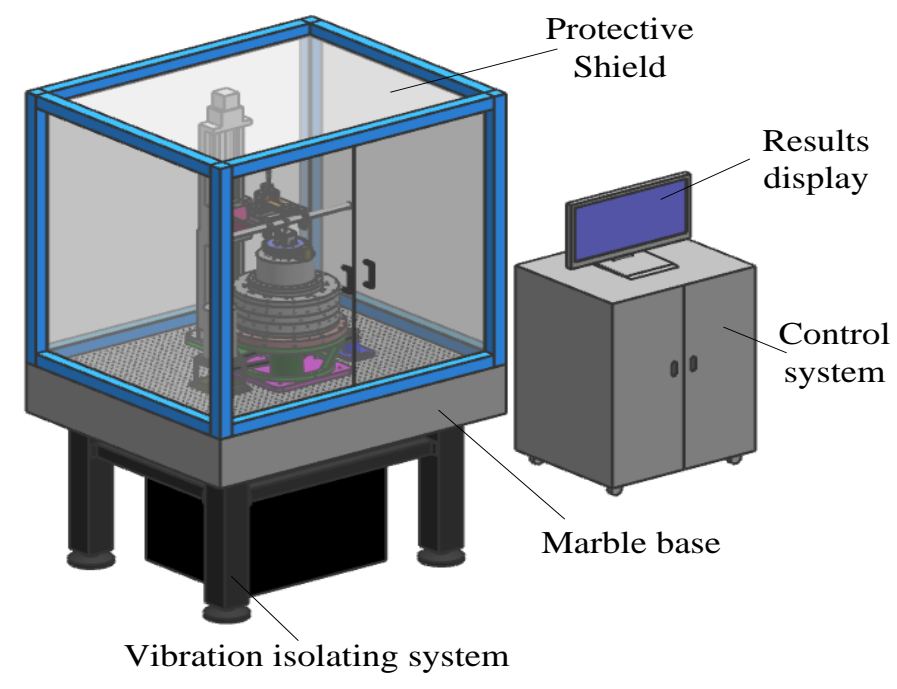

Fig.2 The measuring system for the rotation error of aerostatic ultra-precision spindle

The detail information of measuring platform is shown in Fig.3. Aerostatic ultraprecision spindle was vertically installed on the spindle base. The rotation movement of aerostatic spindle was controlled by the spindle drive. According to the height of aerostatic spindle, the position of displacement sensor in the vertical direction was adjusted by the guideway. The accurate position of displacement sensor was regulated by the 3D displacement adjusting device. The position fixture was used to realize a reverse of displacement sensor. The angle encoder and reading head, which were applied to precisely reverse the artifact, were mounted on the end of aerostatic spindle. The artifact was installed on the eccentric adjusting device, which can be used to reduce the artifact eccentricity deviation from the spindle rotation axis. 


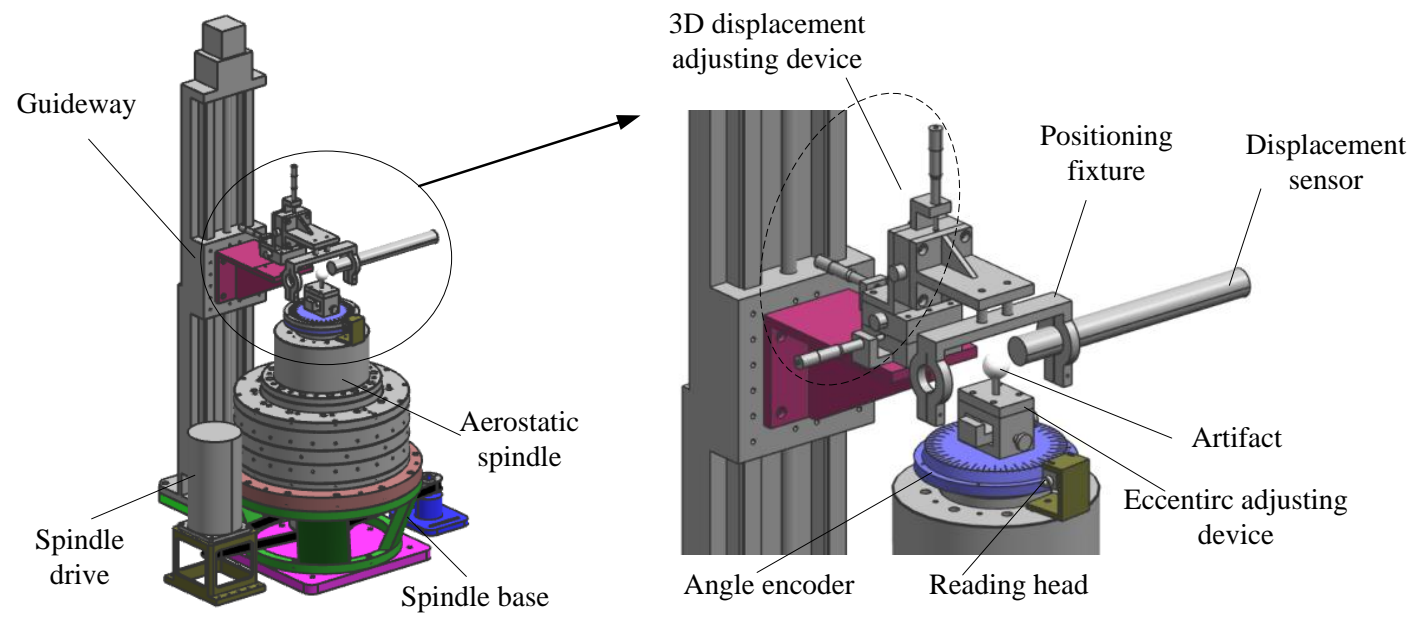

Fig.3 The detail setup of the measuring platform

\section{Error analysis}

Although the Donaldson reversal method is theoretically perfect to error separation, the separation accuracy is still subject to position errors of artifact and sensor, tilt and misalignment error of sensor, artifact eccentricity, spindle axial motion error, angle accuracy of sampling and spindle drive. Since the effect of position errors, tilt and misalignment error has been investigated systematically [19], this study concentrates on the influences of spindle drive, angle accuracy of sampling, artifact eccentricity and spindle axial motion error.

\subsection{Effect of spindle drive}

Both Cappa [19] and Marsh [24] pointed out that the measuring data were obviously affected by the spindle motor and drive. In this study, the comparative experiments were carried out to investigate the effects of the motor drive on the running accuracy of aerostatic ultra-precision spindle. The comparative measuring results between free rotation and motor with the different pole pairs were shown in Fig.4. It can be observed from Fig.4 that the cogging torque of motor had a significantly effect on the results of running accuracy. At the motor pole pairs of 10, the measuring data had 10 peaks and valleys in a circle. The cogging torque of motor can be reduced by increasing of pole pairs, so the rotation error of aerostatic spindle decreased from $0.1752 \mu \mathrm{m}$ to 0.0589 $\mu \mathrm{m}$ with the growth of motor pole pairs from 10 to 64 . However, compared with the measuring results of free rotation without motor drive, the effect of motor drive on the running accuracy cannot be ignored. 


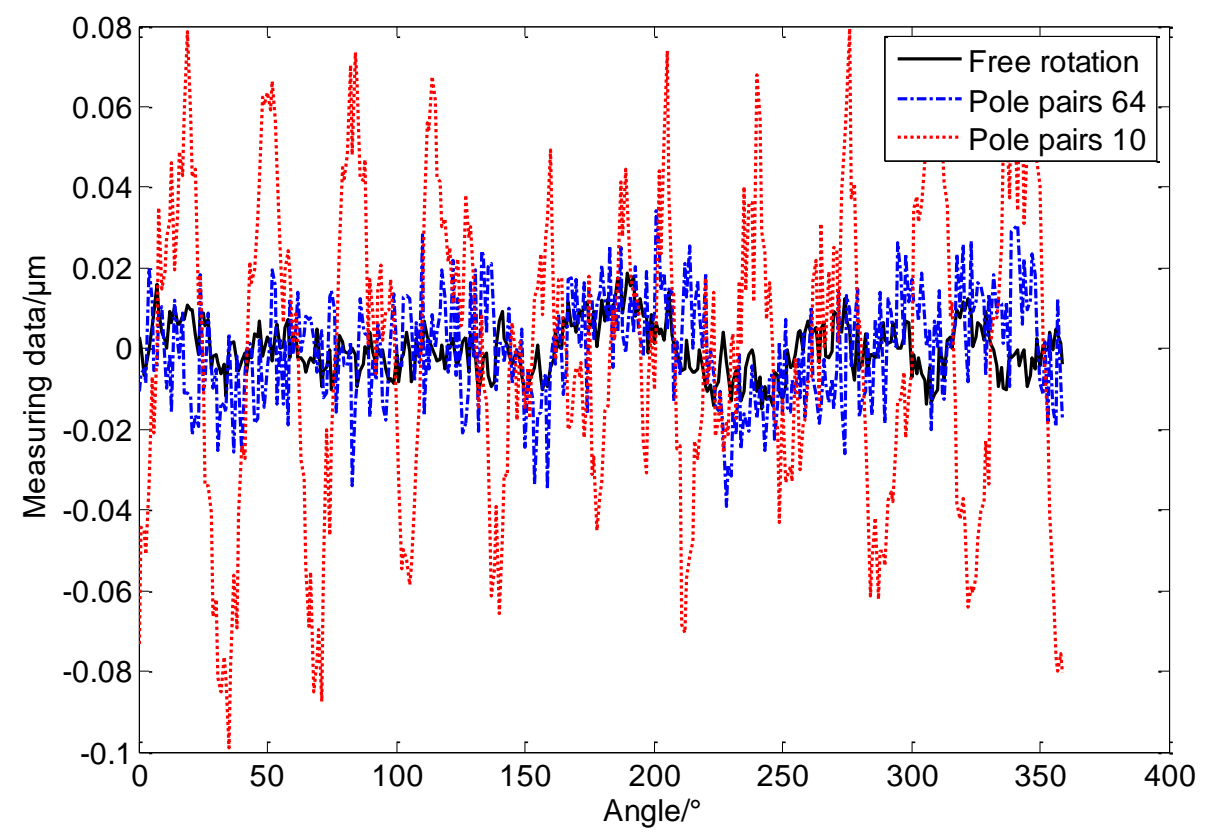

Fig.4 Effect of motor drive on the running accuracy

\subsection{Effect of sampling accuracy}

Ideally, the angle corresponding to measuring displacement should be rigorous equality before and after reverse. However, due to the limit of sampling accuracy, the angle deviation between two measurements was inevitable, as shown in Fig.5.

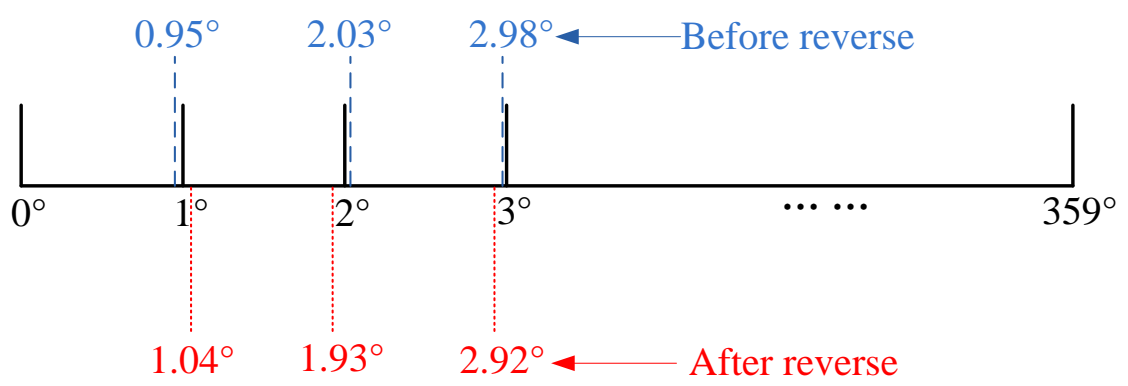

Fig.5 Sampling angle deviation

In this study, an angle correction algorithm was proposed to alleviate the effect of angle deviation on the measuring accuracy. The calculation procedure of the calculation procedure is shown in Fig.6. 


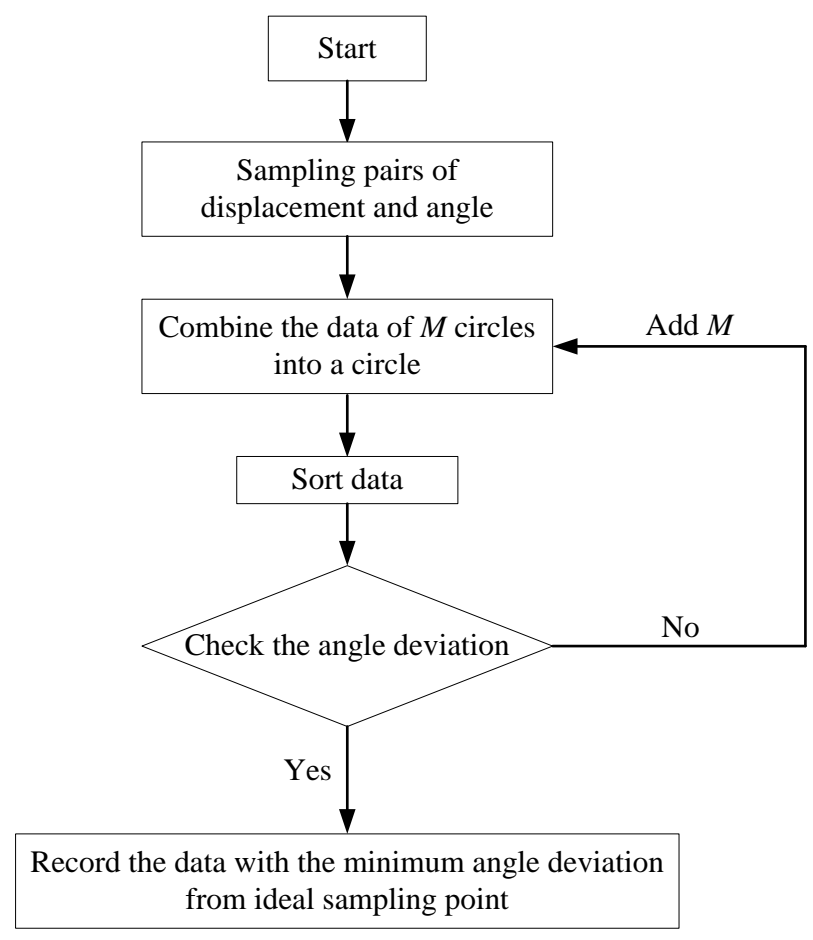

Fig.6 Flow chart of the calculation procedure

In order to avoid the communication delay, the data sampling was triggered by hardware rather than software, and so the displacement and the corresponding angle were recorded simultaneously. The actual sampling angle was recorded by the angular grating, which was mounted on the end of spindle. Due to the limit of sampling accuracy, the angle deviation between actual and ideal sampling point always existed, and the angle deviation of each circle was inconsistent. In order to find the data with the minimum angle deviation from ideal sampling point, the data of $M$ cycles was combined into a circle. The angle deviation can be reduced by increasing the cycle number $M$. The effect of $M$ on the angle deviation is shown in Fig.7. With the increase of $M$ from 1 to 10 , the maximum angle deviation was reduced from $0.298^{\circ}$ to $0.05^{\circ}$. Fig. 8 plots the effect of $M$ on the calculation results of measuring data, which was obtained at the artifact eccentricity of $0.1 \mu \mathrm{m}$. It can be obtained from the Fig. 8 that the calculation results of measuring data were obviously influenced by the deviation angle. The calculation results of rotation error decreased from $61.45 \mathrm{~nm}$ to $48.71 \mathrm{~nm}$ with the growth of $M$ from 1 to 10 . The measuring uncertainty can be reduced by increasing the number of $M$. 


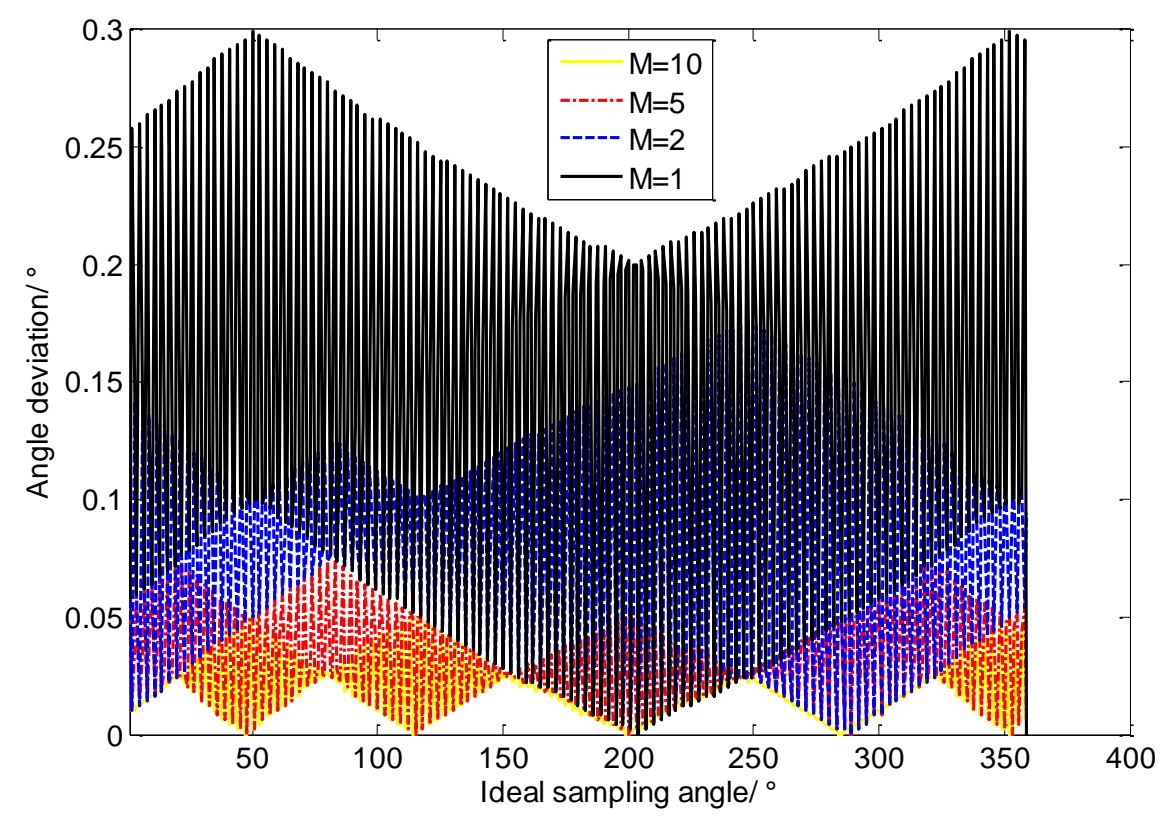

Fig.7 Effect of $M$ on the angle deviation

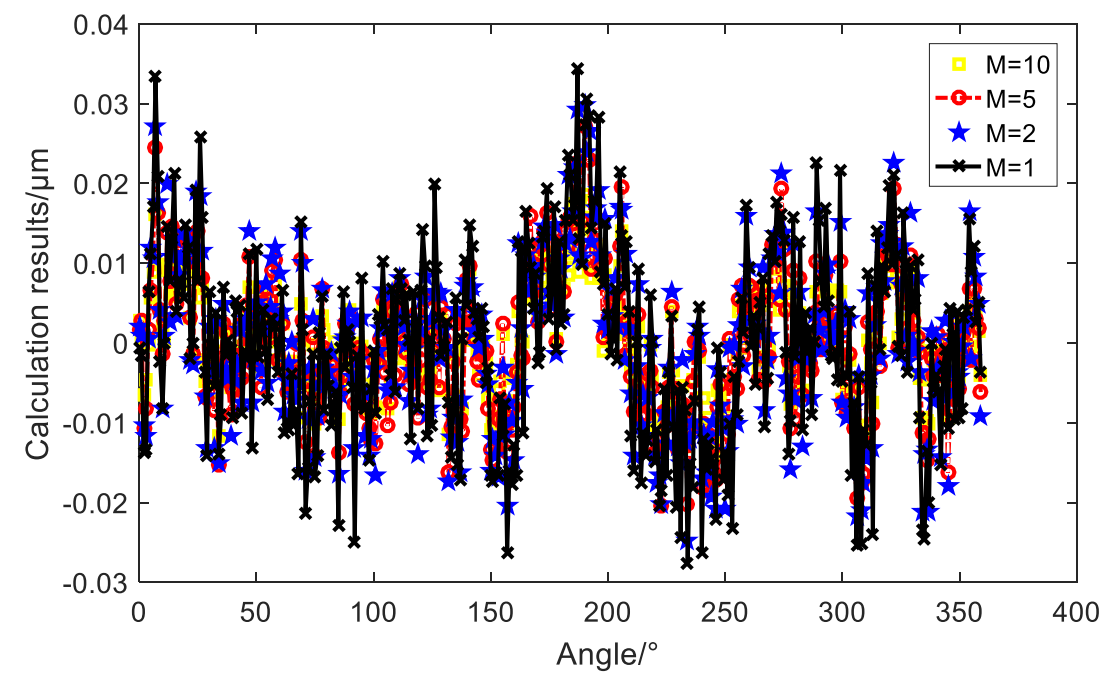

Fig. 8 Effect of $M$ on the calculation results

\subsection{Effect of artifact eccentricity}

Cappa et al. [19] pointed out that the artifact eccentricity of $10 \mu \mathrm{m}$ resulted in the measurement errors of $4 \mathrm{~nm}$ at the artifact radius of $12.7 \mathrm{~mm}$. Therefore, in this paper, the method of harmonic analysis was applied to investigate the effect of artifact eccentricity on the measurement results. The schematic of artifact eccentricity is shown in Fig.9. The rotation axis of spindle is located at $O_{1}$. The center of artifact is $O$, which varies with rotation angle $\alpha$. The measurement data obtained from the displacement sensor can be expressed as:

$$
d=d_{0}-d_{1}
$$


where $d$ is the measurement data, $d_{0}$ is the distance from spindle rotation axis to sensor, $d_{l}$ is the distance variable which varies with rotation angle $\alpha$, and can be denoted as:

$$
d_{1}=e \sin \alpha+\sqrt{r^{2}-(l-e \cos \alpha)^{2}}
$$

where $e$ is the artifact eccentricity, $r$ the radius of the artifact, $l$ is the sensor position deviation from spindle rotation axis. Fig.9(b) plots the condition of sensor without position deviation, and Eq.(6) can be rewritten as:

$$
d_{1}=e \sin \alpha+\sqrt{r^{2}-(e \cos \alpha)^{2}}
$$

Eq.(7) is different from Eq. (63) in literature [19], at the relative small artifact eccentricity, the calculated results difference between the two equation can be ignored.

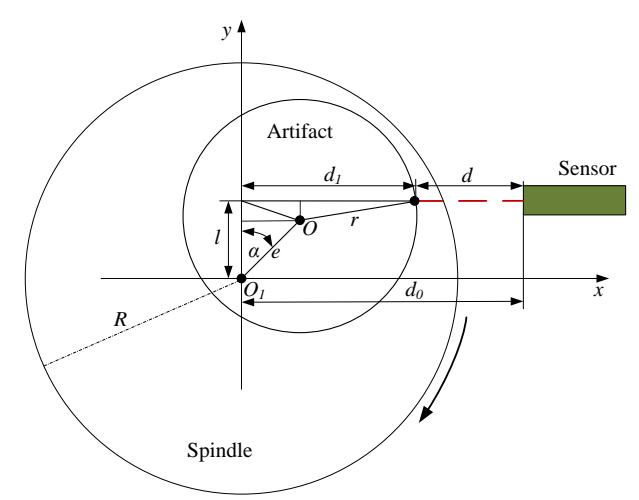

(a)

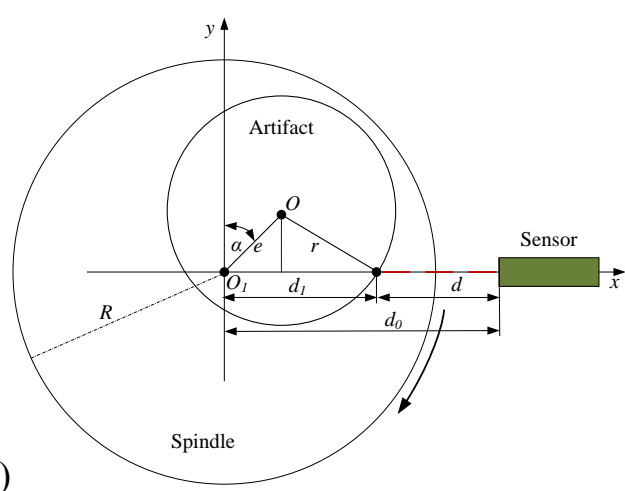

(b)

Fig.9 Schematic of artifact eccentricity effect: Sensor with (a) and (b) without position deviation

At the artifact radius of $15 \mathrm{~mm}$, the calculation results of Eq.(7) at the different artifact eccentricity is shown in Fig.10. It can be observed from the Fig.10 that the artifact eccentricity had a significant influence on the calculation results. The rotation errors increased obviously with the growth of artifact eccentricity. 


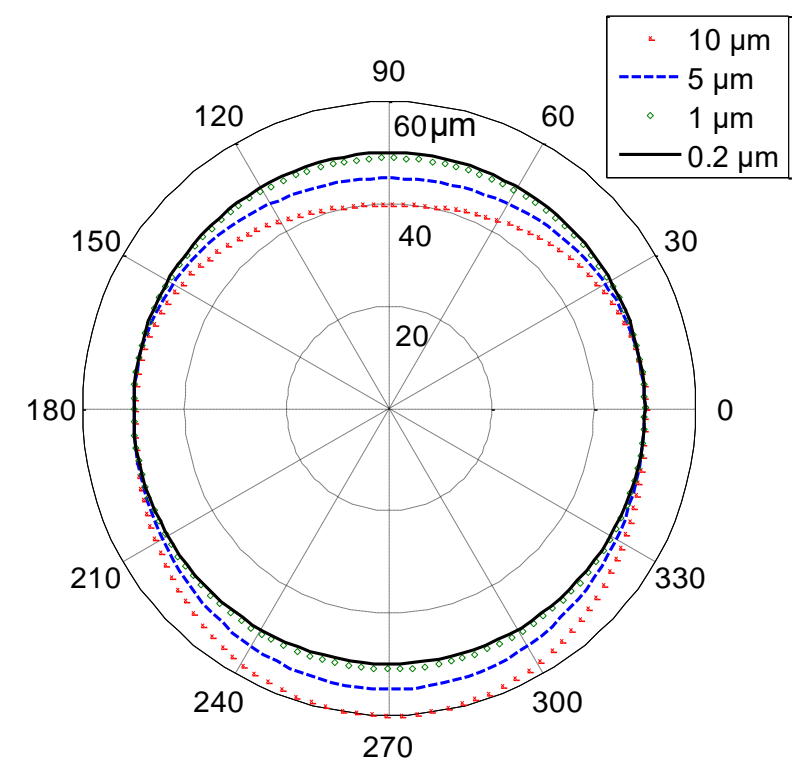

Fig.10 Calculation results at the different artifact eccentricity

A single circle of the rotary error data is a random signal, but multiple cycles are repetitive signals. According to Fourier theorem, the repetitive signals can be expressed as a sum of different order harmonic. Based on the residual equation:

$$
\Delta d\left(\alpha_{i}\right)=d\left(\alpha_{i}\right)-\frac{1}{N} \sum_{i=0}^{N-1} d\left(\alpha_{i}\right)
$$

Fourier coefficient can be denoted as

$$
\begin{aligned}
& a_{k}=\frac{2}{N}\left[\sum_{i=0}^{N-1} \Delta d\left(\alpha_{i}\right) \cdot \cos \left(k \alpha_{i}\right)\right] \\
& b_{k}=\frac{2}{N}\left[\sum_{i=0}^{N-1} \Delta d\left(\alpha_{i}\right) \cdot \sin \left(k \alpha_{i}\right)\right]
\end{aligned}
$$

where $N$ is the measuring points, $k$ is the order of the harmonic. Therefore, in the polar coordinates, the rotation error can be expressed as:

$$
\rho(\alpha)=r_{o}+\sum_{k=1}^{\infty} a_{k} \cos k \alpha+\sum_{k=1}^{\infty} b_{k} \sin k \alpha
$$

where $r_{o}$ is the reference radius. The different harmonic of rotation error can be calculated by the Eq.(11). In this study, at the artifact eccentricity of $10 \mu \mathrm{m}$, the measuring data obtained from displacement sensor is shown Fig.11, and the harmonics 
with the order from 1 to 6 are shown Fig. 12 .

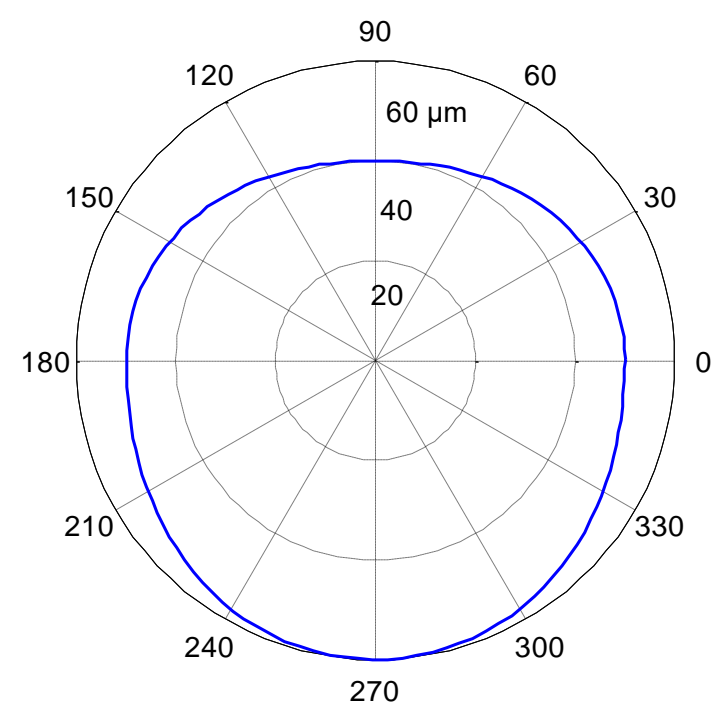

Fig. 11 Measuring results at the artifact eccentricity of $10 \mu \mathrm{m}$

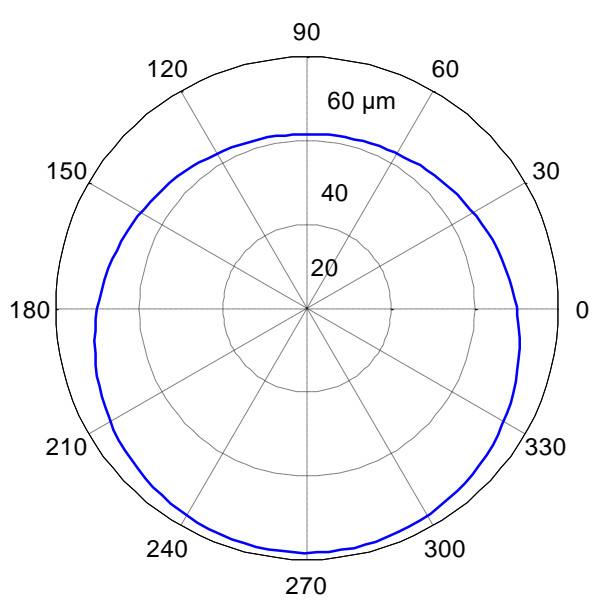

(a) First order

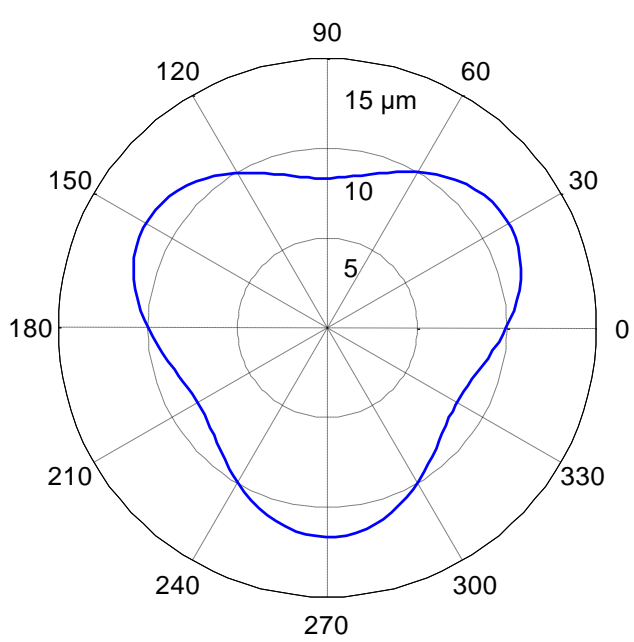

(c) Third order

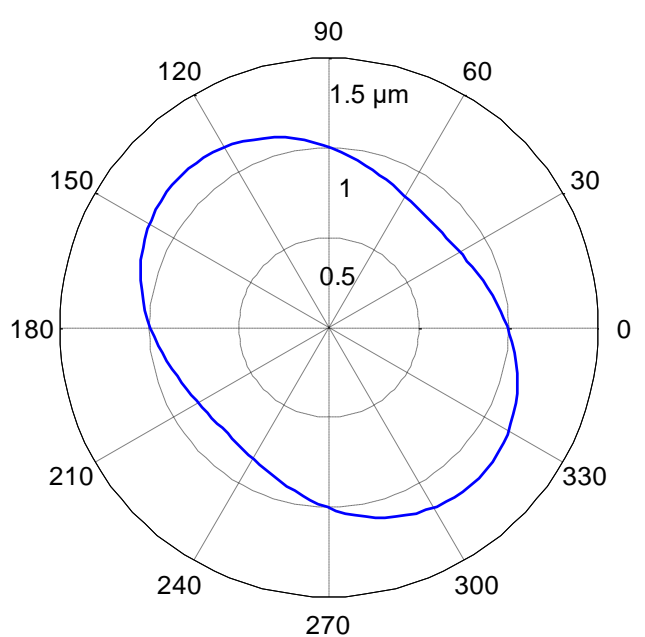

(b) Second order

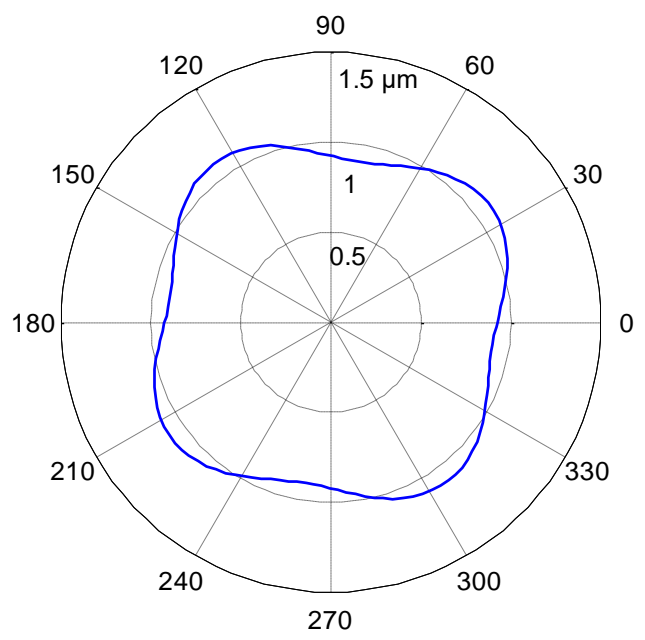

(d) Fourth order 


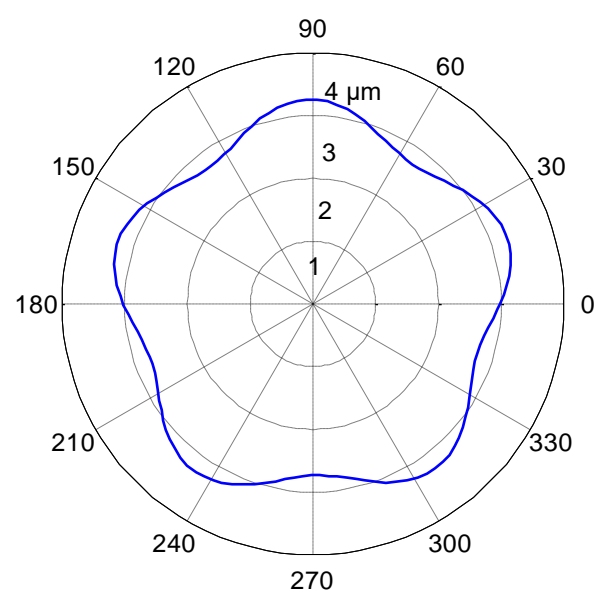

(e) Fifth order

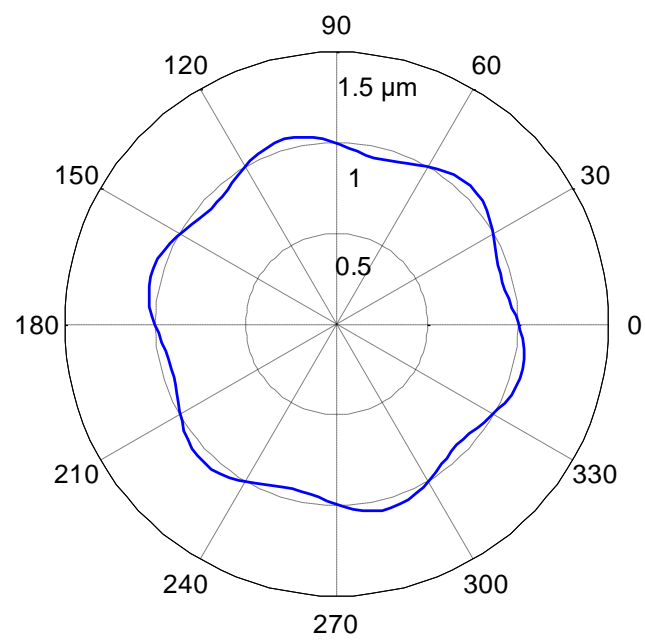

(f) Sixth order

Fig.12 Effect of artifact eccentricity of $10 \mu \mathrm{m}$ at the different harmonic

It can be observed from Fig.11 and Fig.12 that the track of measuring results was significantly changed by the artifact eccentricity. The amplitude of odd harmonic was obvious bigger than even harmonic. For either odd or even harmonic, the growth of the order resulted in the reduction of harmonic amplitude.

In order to alleviate the measuring uncertainty, the artifact eccentricity should be controlled as small as possible. Fig. 13 plots the spindle rotation error at the artifact eccentricity of $0.1 \mu \mathrm{m}$, and the harmonics with the order from 1 to 6 are shown Fig.14.

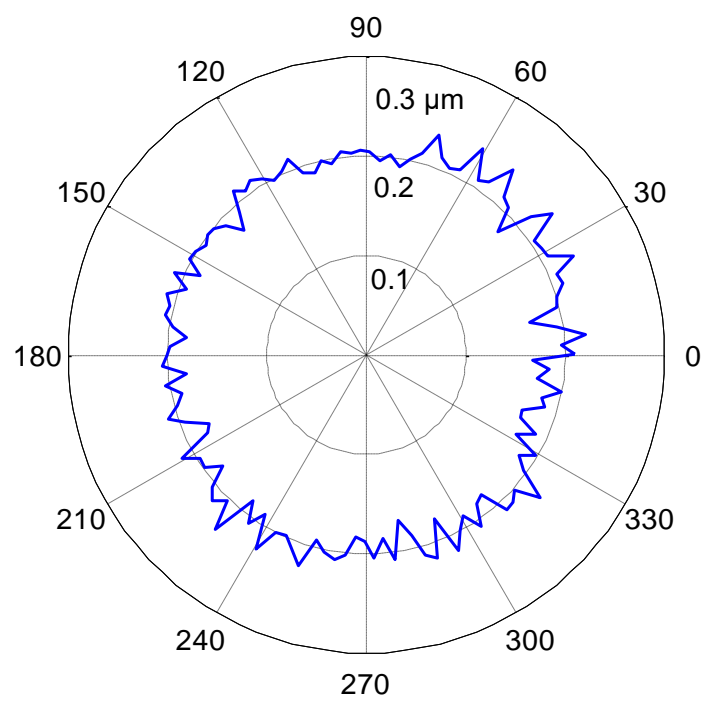

Fig. 13 Measuring results at the artifact eccentricity of $0.1 \mu \mathrm{m}$ 


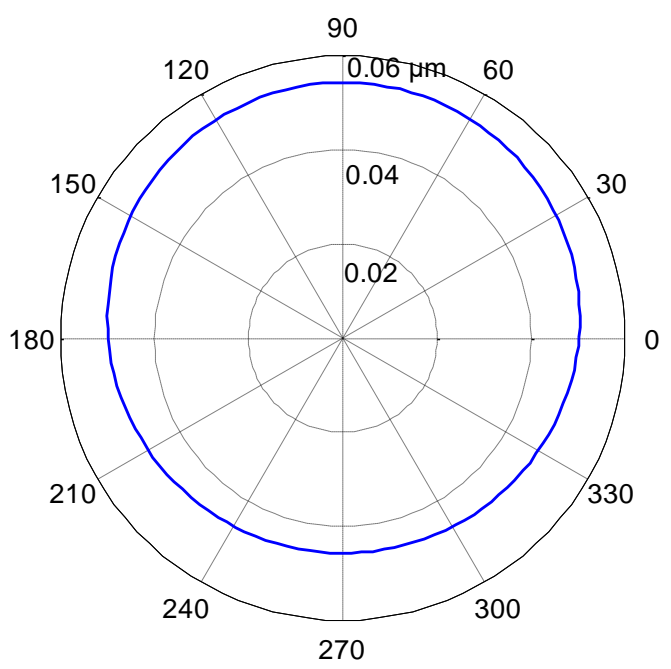

(a) First order

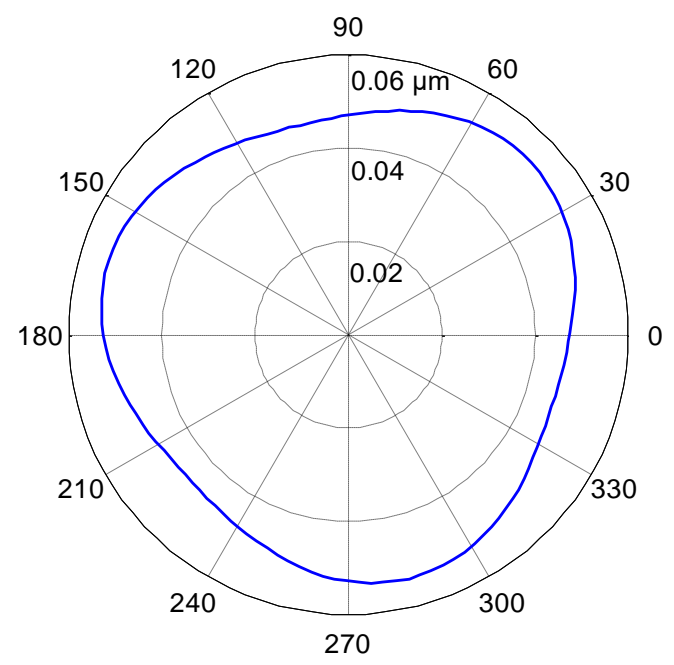

(c) Third order

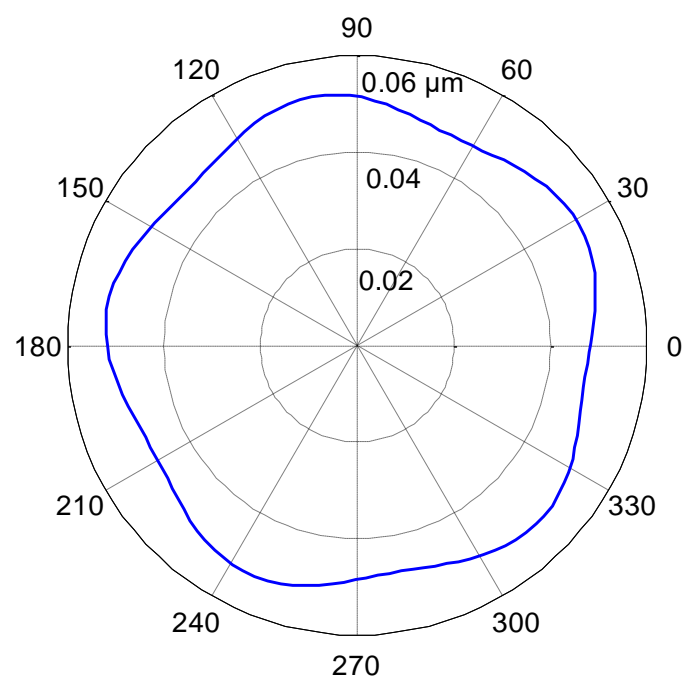

(e) Fifth order

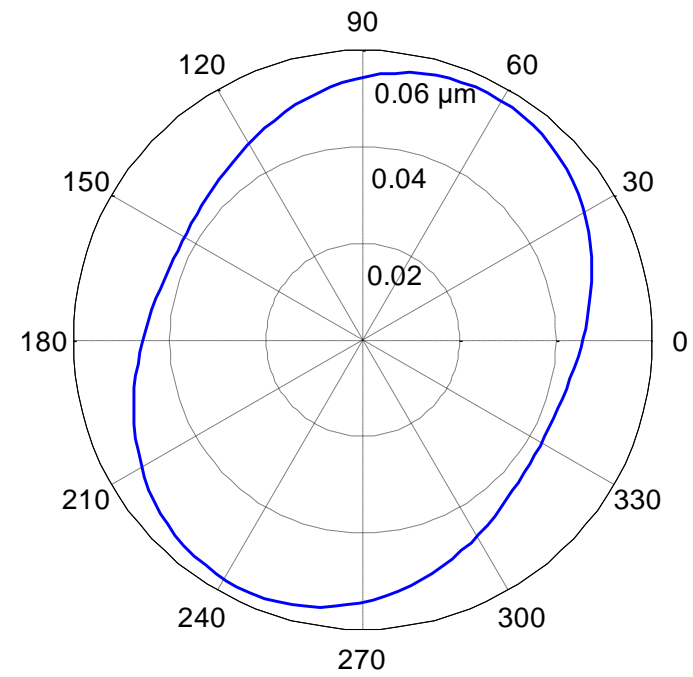

(b) Second order

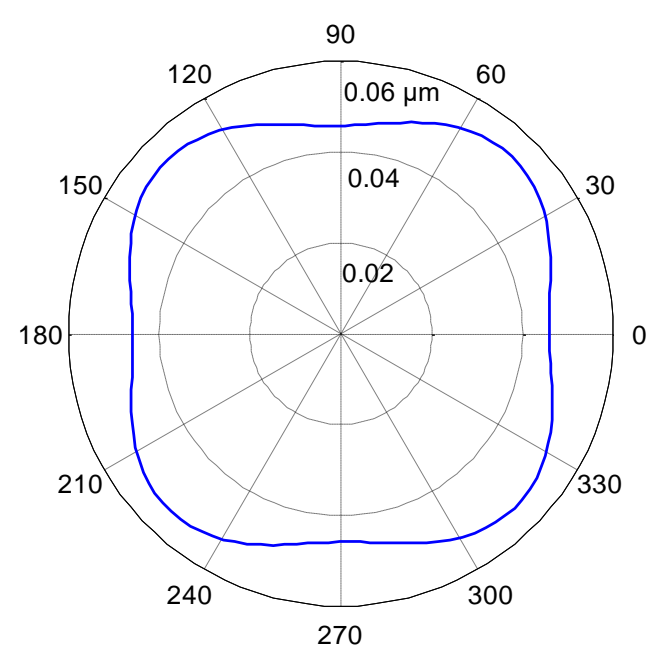

(d) Fourth order

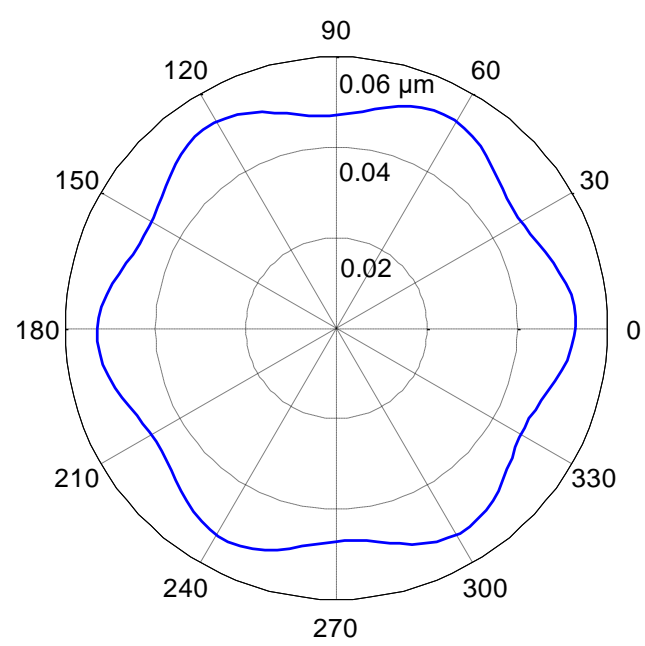

(f) Sixth order

Fig.14 Effect of artifact eccentricity of $0.1 \mu \mathrm{m}$ at the different harmonic 
Compared with Fig.11, it can be seen from Fig.13 that the measuring accuracy of rotation error can be significantly improved by improving the artifact eccentricity. However, considering the limit of apparatus, the artifact eccentricity always existed in the measurement process. At artifact eccentricity of $10 \mu \mathrm{m}$ and $0.1 \mu \mathrm{m}$, the track of first harmonic was a circle with the radius of artifact eccentricity, as shown in Fig.12(a) and Fig.14(a), respectively. The first harmonic was caused by artifact eccentricity. Therefore, the first harmonic of measuring data can be eliminated to reduce the uncertainty, as denoted by Eq.(12).

$$
\rho^{\prime}(\alpha)=\rho(\alpha)-\left[a_{1} \cos \alpha+b_{1} \sin \alpha\right]
$$

At the artifact eccentricity of $10 \mu \mathrm{m}$, Fig.15(a) plots the measuring data with and without the first harmonic. Compared with the original measuring data, the data eliminated the first harmonic was more close to the original measuring data at the artifact eccentricity of $0.1 \mu \mathrm{m}$. The measuring data with and without the first harmonic at the artifact eccentricity of $0.1 \mu \mathrm{m}$ is shown Fig.15(b). It can be observed from Fig.15(b) that the eliminated data was more close to the figure center than original data, which means the accuracy of measuring results can be improved by eliminating the first harmonic.

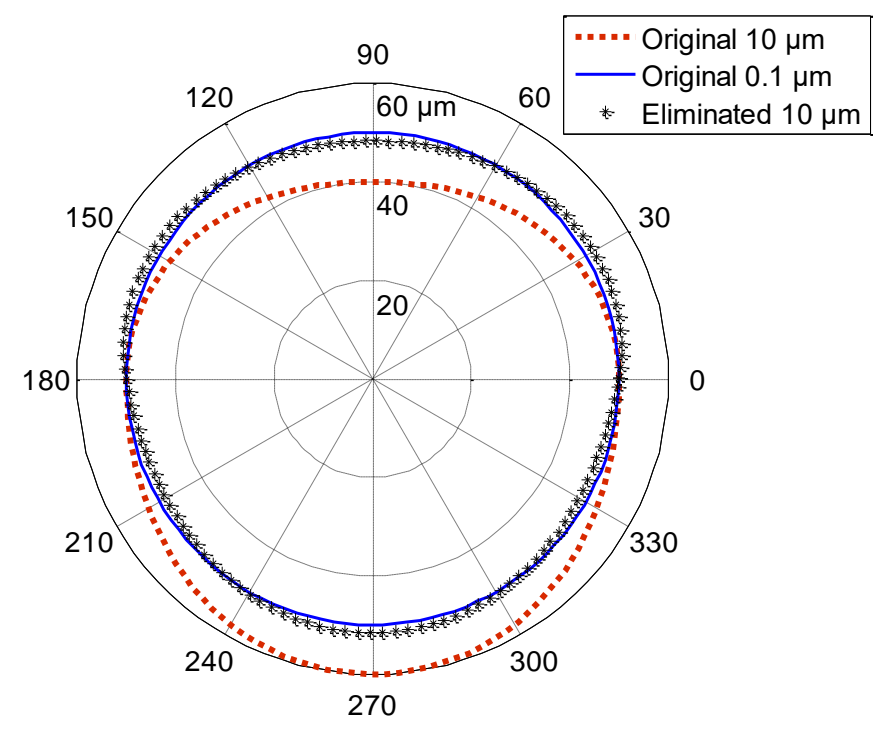

(a) Artifact eccentricity of $10 \mu \mathrm{m}$ 


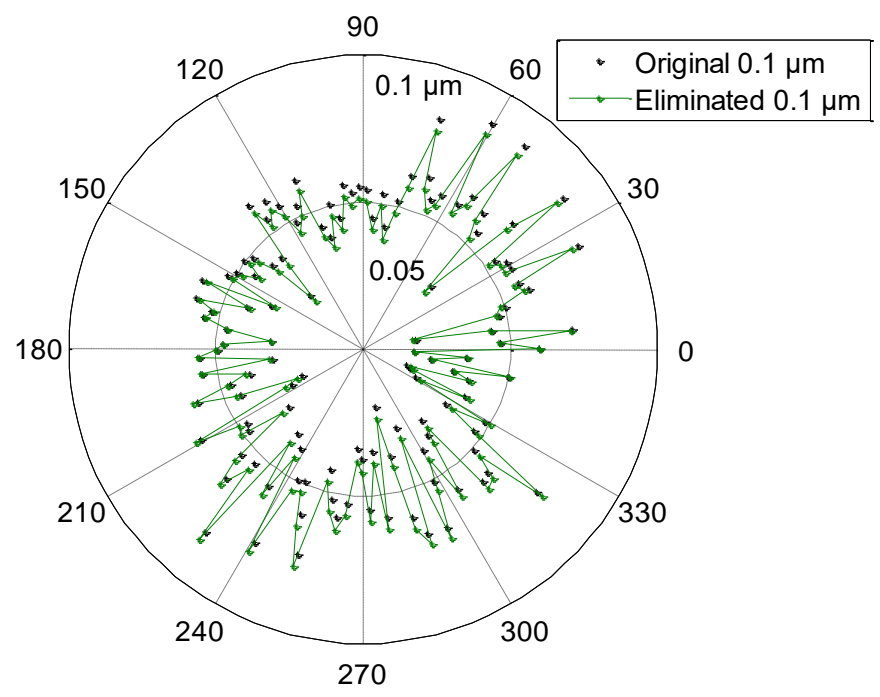

(b) Artifact eccentricity of $0.1 \mu \mathrm{m}$

Fig.15 Eliminate the first harmonic of rotation error

\subsection{Effect of axial motion}

The axial motion of spindle inevitably exists in the rotation error measuring process. The axial motion effect on the measuring result is shown Fig.16. The relationship between the axial motion $\Delta h$ and measuring error $\Delta d$ can be denoted as:

$$
\Delta d=r-\sqrt{r^{2}-\Delta h^{2}}
$$

Generally, the air film thickness of aerostatic spindle is less than $10 \mu \mathrm{m}$. At the artifact radius of $20 \mathrm{~mm}$, the maximum axial motion of $10 \mu \mathrm{m}$, the measuring error calculated by Eq.(12) is $2.5 \mathrm{~nm}$. In the operating condition, the spindle axial motion is less than $0.2 \mu \mathrm{m}$ in most case, so the effect of axial motion on measuring result can be ignored.

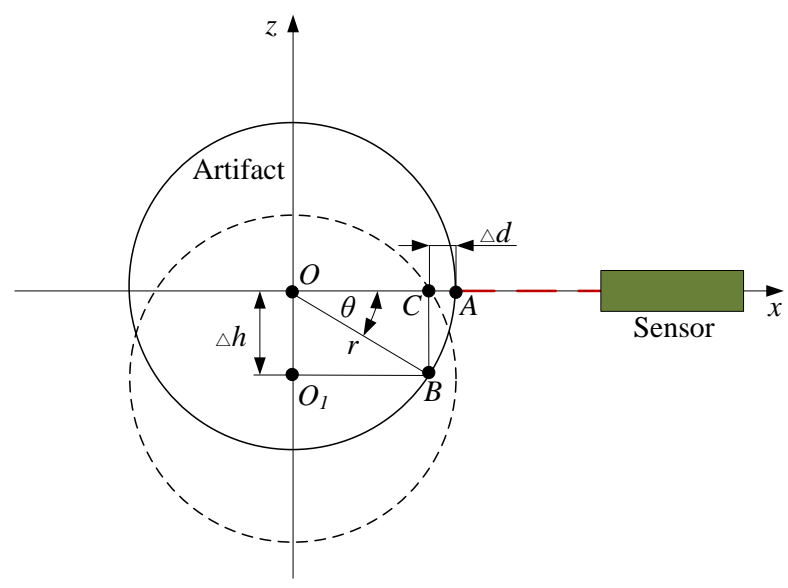

Fig.16 Schematic of axial motion effect 


\section{Results and discussion}

\subsection{Measuring process and results}

The geometric structure of aerostatic ultra-precision spindle is shown in Fig.17. The machining and measurement accuracy is significantly influenced by the radial motion error of the spindle when applied to machine tools and metrology instruments, which is mainly determined by the manufacturing errors, design parameters and operating conditions $[2,4]$. Accurate measurement of rotation error can provide useful guidelines for the design and manufacture of aerostatic ultra-precision spindle, as well as the improvement in functional accuracy when it is used in the instrument and equipment.

Based on the error analysis discussed above, a nanometer system for measuring the rotation error of aerostatic ultra-precision spindle was developed, as shown in Fig.18. The angle encoder and reading head was applied to precisely control the reverse of artifact with the precision of $0.1^{\circ}$. The reverse of displacement sensor was realized by the positioning fixture. Because the measuring accuracy was influenced by the positioning error of artifact and sensor, the manufacturing and assembling errors of positioning fixture and eccentric adjusting device was controlled within $0.2 \mu \mathrm{m}$. And the axial motion of aerostatic spindle was limited to $0.1 \mu \mathrm{m}$, so the effect of axial motion errors on measuring results can be ignored. The detail information of the experiment platform is listed in Tab.1. 


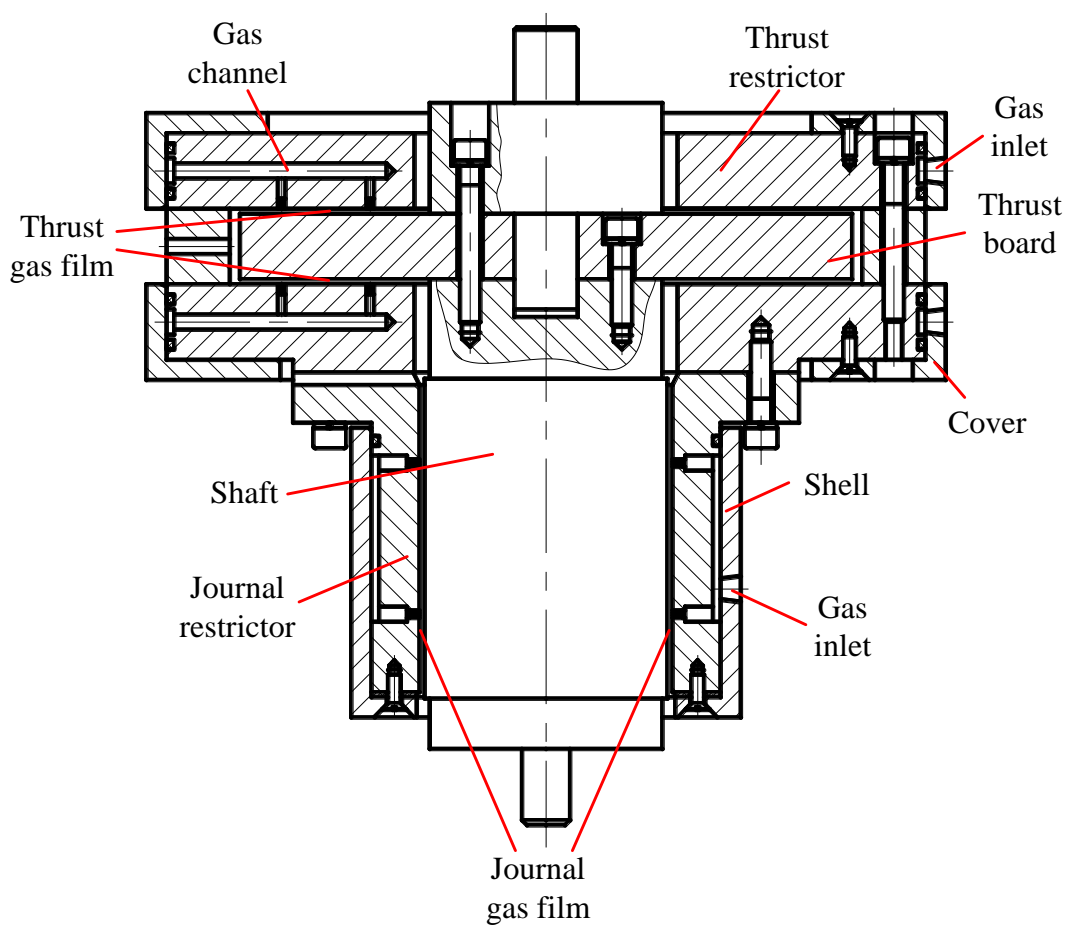

Fig.17. Structure of aerostatic spindle
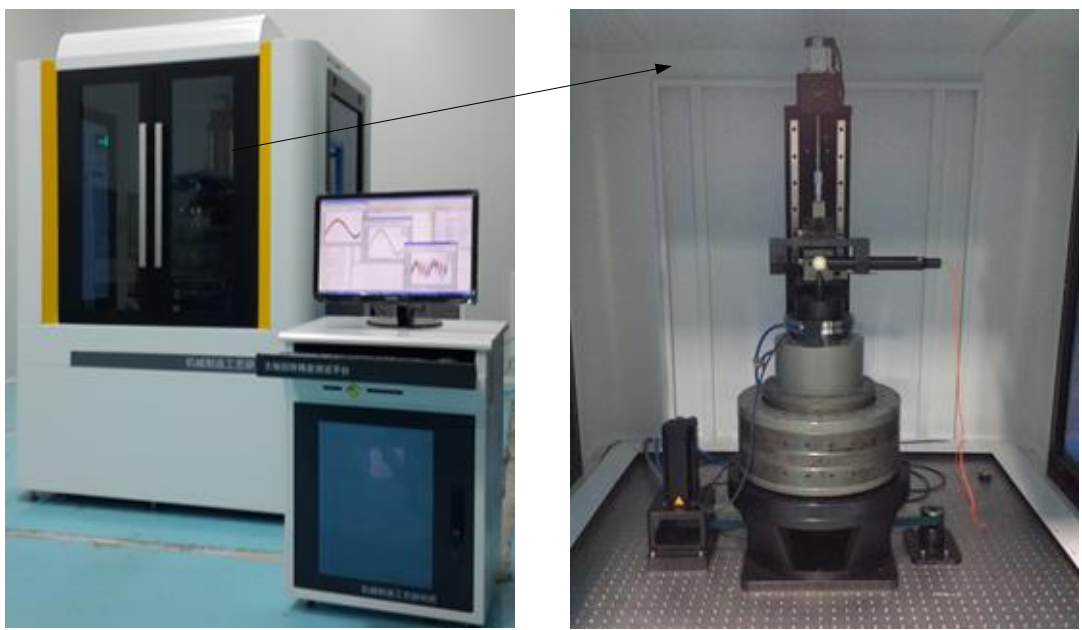

Fig.18 Experiments platform for rotation error of aerostatic ultra-precision spindle

The eccentricity of artifact was limited within $0.1 \mu \mathrm{m}$, as shown in Fig.19. During the measuring process, the protective shield was closed to alleviate the effects of environmental noise and airflow. In order to reduce the sensor temperature drift, the fluctuation of surrounding circumstance temperature was controlled within $0.1^{\circ} \mathrm{C}$. Before the measuring system started to record the displacement and the corresponding angle data, the displacement sensor is on operation at least 30 minutes. During the data collection process, the power of spindle motor was cut off to eliminate the influence of motor drive. 
Tab.1 The detail information of the experiment platform

\begin{tabular}{ll}
\hline Supply pressure (bar) & 5 \\
Resolution of sensor $(\mathrm{nm})(\mathrm{STIL}$ CL0-MG210) & 1.5 \\
Sampling frequency of sensor (Hz) (STIL CL0-MG210) & $100 \sim 2000$ \\
Surrounding temperature $\left(^{\circ}\right)$ & $20 \pm 0.1$ \\
Roundness of artifact $(\mathrm{Nominal})(\mathrm{nm})(\mathrm{Mahr})$ & 32 \\
Diameter of artifact $(\mathrm{mm})(\mathrm{Mahr})$ & 15 \\
Fixture misalignment $(\mu \mathrm{m})$ & 0.2 \\
Fixture Tilt ( $\mu \mathrm{m})$ & 0.15 \\
Angle encoder roundness $(\mu \mathrm{m})$ & 0.1 \\
Assembling parallelism errors $(\mu \mathrm{m})$ & 0.2 \\
Assembling verticality errors $(\mu \mathrm{m})$ & 0.2 \\
Sampling frequency $(\mathrm{Hz})$ & 600 \\
Free rotation speed $(\mathrm{RPM})$ & 50 \\
\hline
\end{tabular}

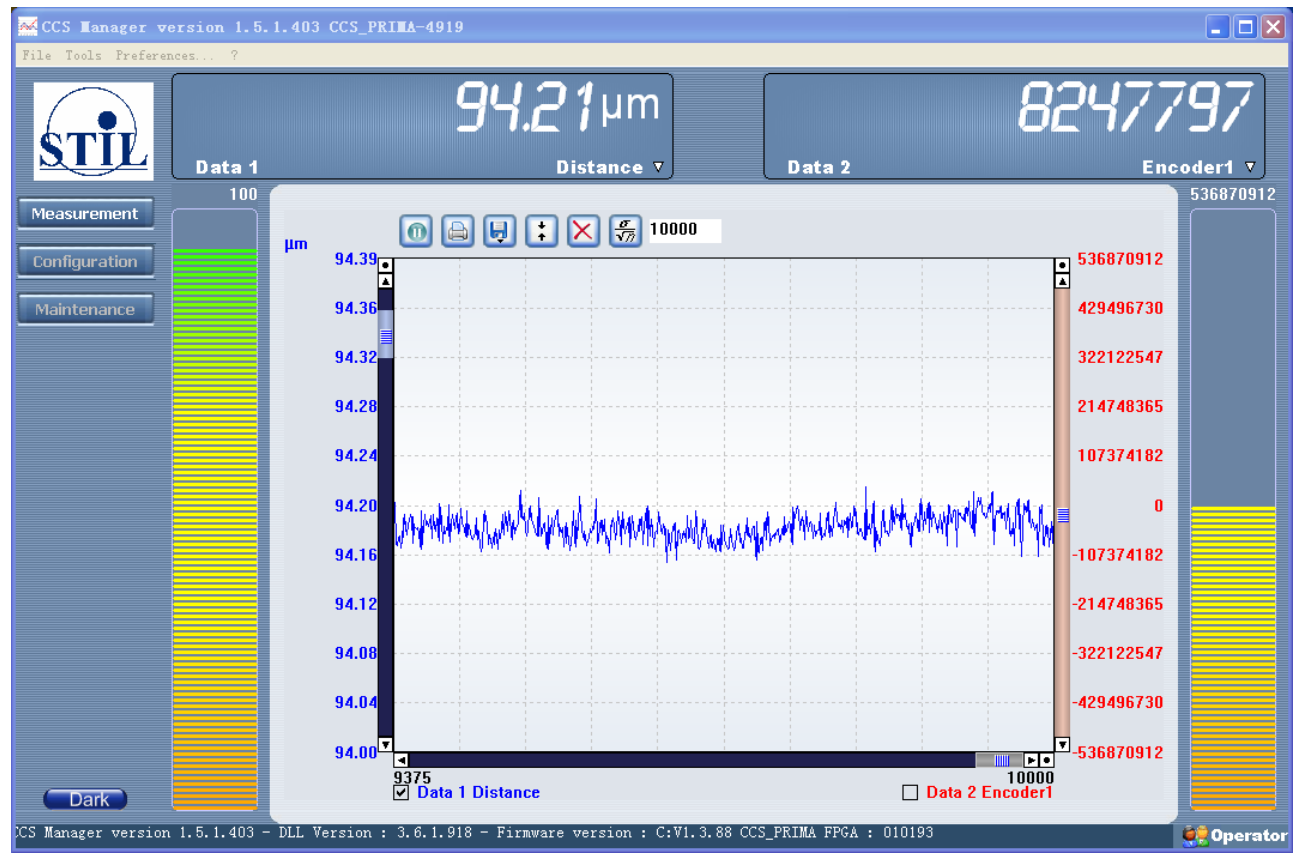

Fig.19 Artifact eccentricity

Fig.20 plots the original data with 25 cycles obtained by the displacement sensor. Based on the angle correction algorithm proposed in this paper, the original measuring data of 25 cycles was combined into a circle. The angle deviation is reduced from $0.298^{\circ}$ to $0.0167^{\circ}$, as shown in Fig. 21 . 


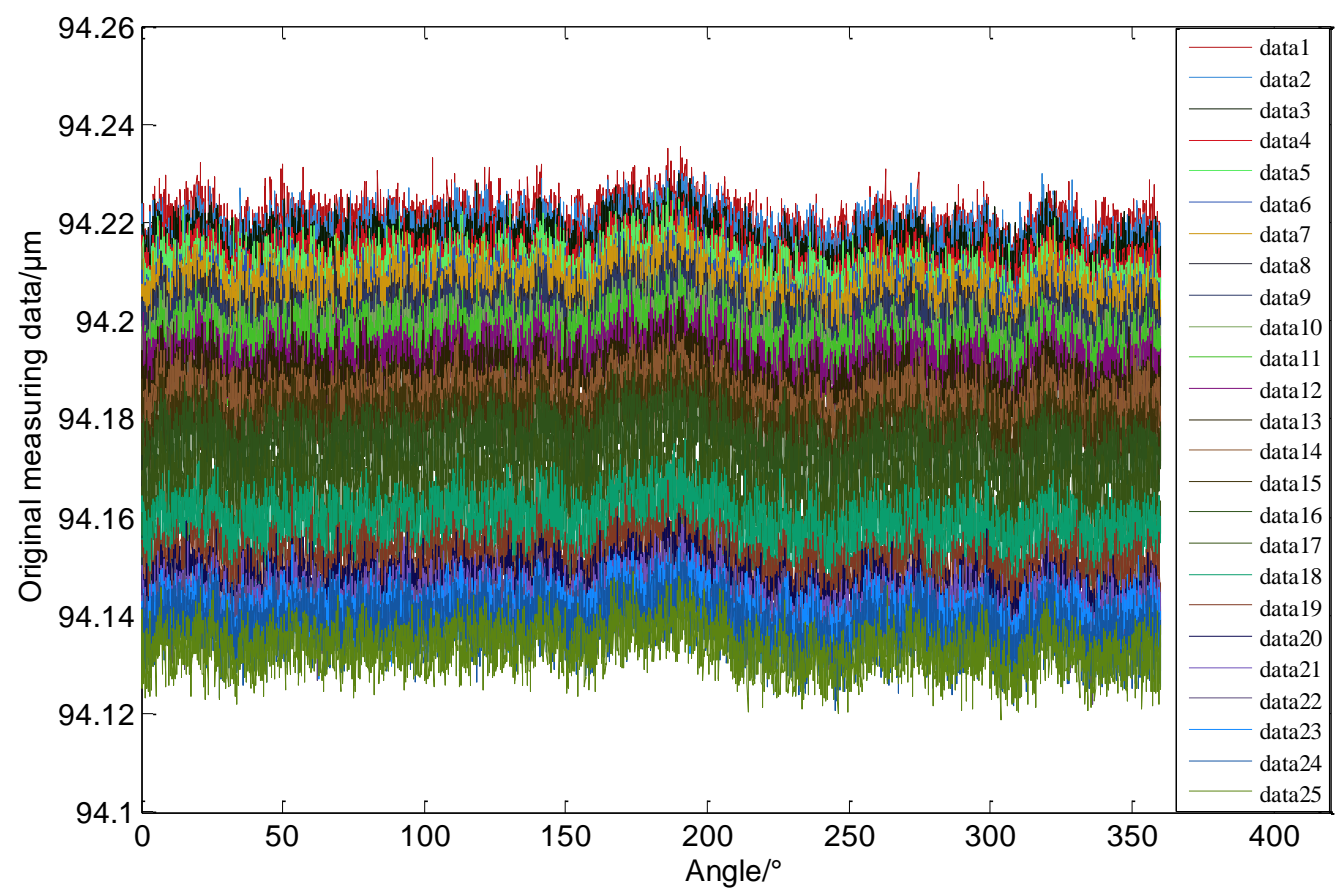

Fig.20 plots the original data with 25 cycles

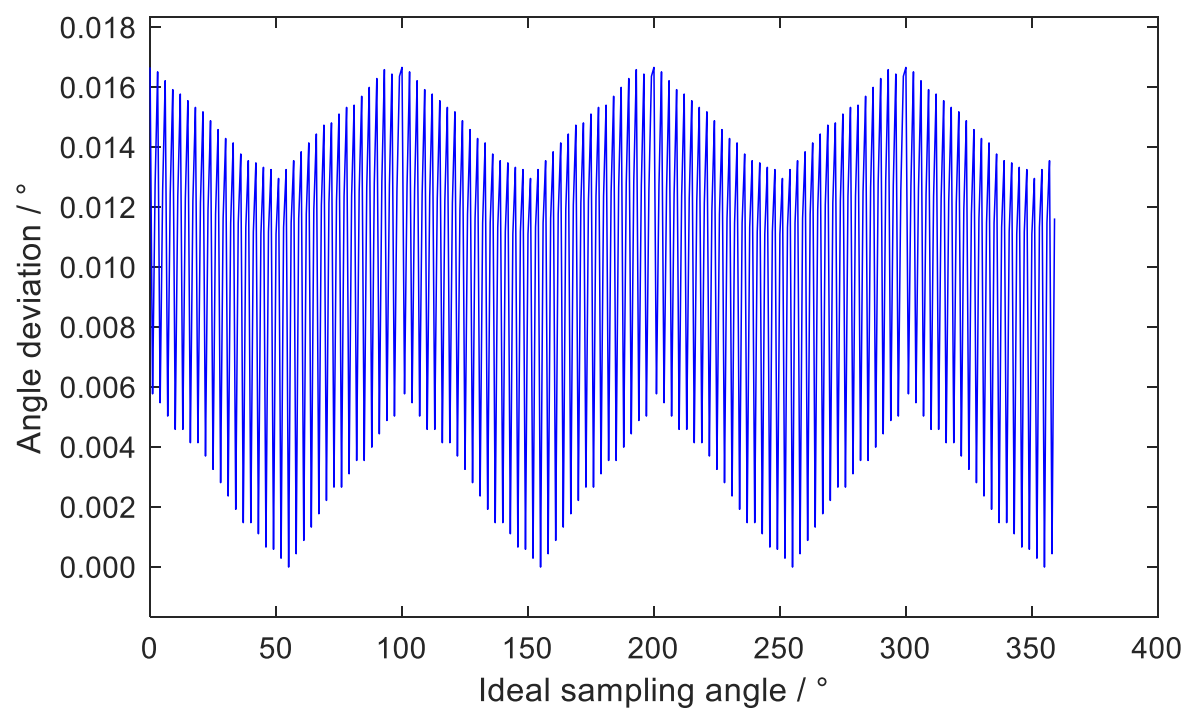

Fig.21 Angle deviation of combined data

Applying Eq.(12) to the combined data, the before and after reverse rotation errors are shown in Fig.22. Based on the combined data, as shown in Fig.21, the rotation error of aerostatic ultra-precision spindle and the roundness of artifact can be obtained by solving Eq.(3) and Eq.(4), as shown in Fig.23 and Fig.24, respectively. 


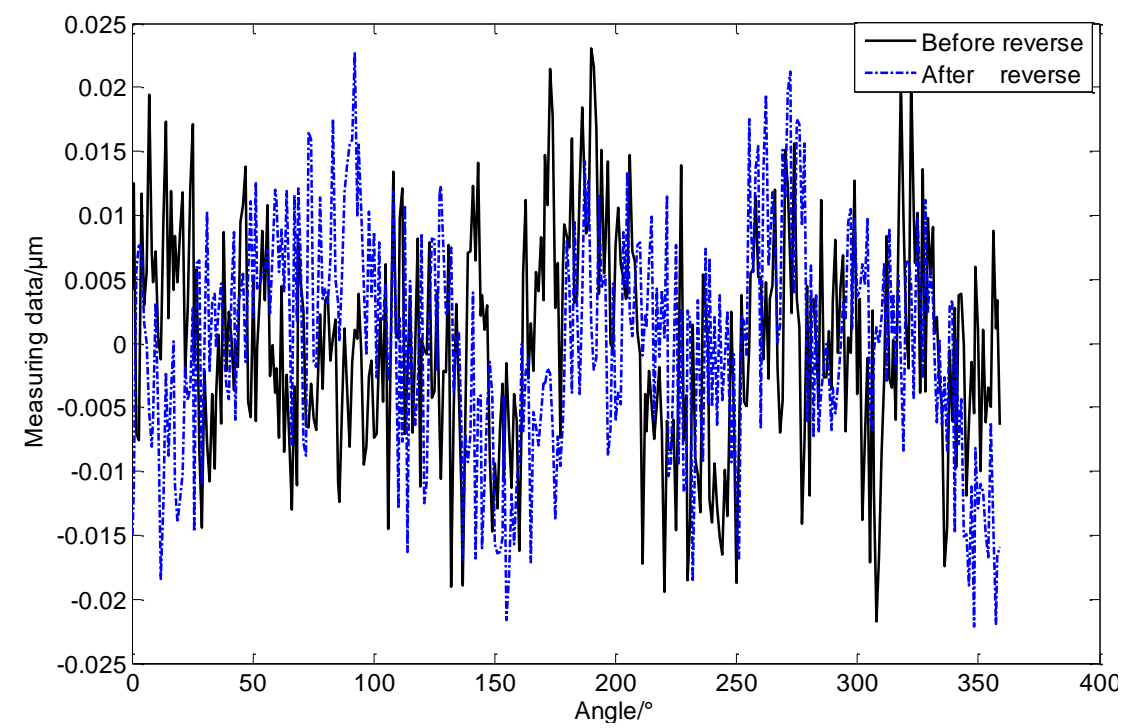

Fig.22 The Combined measuring data

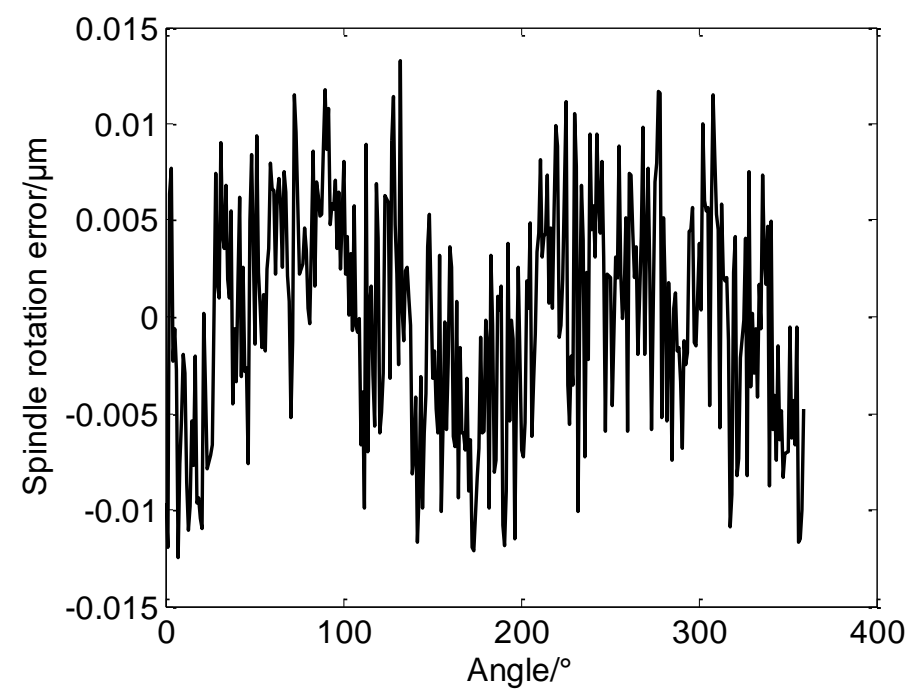

Fig.23 The measuring results of spindle rotation error

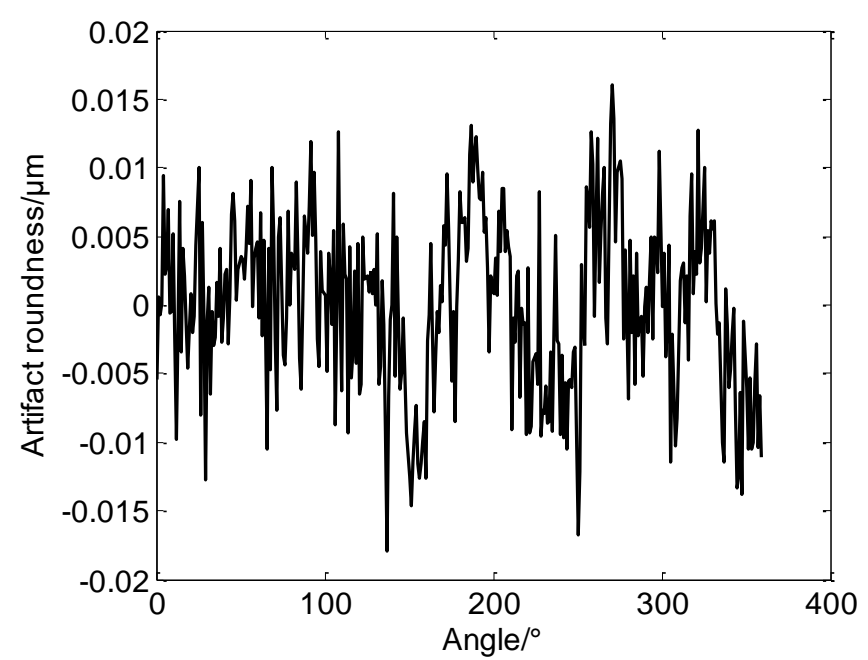

Fig.24 The measuring results of artifact roundness 


\subsection{Measuring uncertainty}

The measuring results consist of the synchronous error and asynchronous error. The synchronous error can be obtained by averaging 10 groups of the combined consecutive measuring data, and the value of synchronous error was determined by calculating the peak-to-valley value of the 720 points in the average of 10 groups of the combined data. The method to determine the asynchronous error was different from the synchronous error. The value of asynchronous error was achieved from the peak-to-valley value with 250 cycles of the spindle rotation, which presented all 180000 measuring points were considered into the calculation. The repeatability of measuring results without reverse is listed in Tab.2. Because of the influence of temperature and pressure fluctuation, electrical noise and structural vibration, the uncertainty of the asynchronous error was obvious bigger than the value of synchronous error, as denoted by standard deviation in Tab. 2.

Tab.2 The repeatability of measuring results

\begin{tabular}{ccc}
\hline Number & Synchronous error $(\mathrm{nm})$ & Asynchronous error $(\mathrm{nm})$ \\
\hline 1 & 43.8 & 3.6 \\
2 & 43.7 & 2.7 \\
3 & 43.9 & 4.1 \\
4 & 44.0 & 3.7 \\
5 & 43.7 & 2.9 \\
6 & 43.9 & 3.5 \\
7 & 43.8 & 4.2 \\
8 & 43.7 & 3.3 \\
9 & 43.8 & 3.6 \\
10 & 43.8 & 2.9 \\
\hline Mean & 43.81 & 3.45 \\
Standard deviation & 0.0994 & 0.5039 \\
\hline
\end{tabular}

The repeatability validation of the modified Donaldson reversal method is listed in Tab.3. It can be observed from Tab.3 that the uncertainty of both the spindle rotation error and artifact form error can be controlled in nanometer level. Besides, the separated value of the artifact form error is very close to the nominal roundness of $32 \mathrm{~nm}$. 
However, the uncertainty of measuring results in this study was still bigger than the Cappa and Marsh's results [19, 24]. The resolution of displacement sensor and the roundness of artifact were the two major reasons for the uncertainty of measuring results.

Tab.3 The repeatability of spindle rotation error and artifact form error

\begin{tabular}{ccc}
\hline Number & $\begin{array}{c}\text { Spindle rotation error } \\
(\mathrm{nm})\end{array}$ & Artifact form error (nm) \\
\hline 1 & 28.3 & 31.4 \\
2 & 27.6 & 32.6 \\
3 & 28.4 & 31.8 \\
4 & 29.1 & 33.3 \\
5 & 30.2 & 32.1 \\
6 & 29.3 & 30.9 \\
7 & 28.7 & 33.5 \\
8 & 27.5 & 33.8 \\
9 & 28.9 & 32.4 \\
10 & 26.9 & 31.5 \\
\hline Mean & 28.49 & 32.33 \\
\hline Standard deviation & 0.9723 & 0.9707 \\
\hline
\end{tabular}

\section{Conclusions}

Based on the Donaldson reversal method, a nanometer system for measuring the rotation error of aerostatic ultra-precision spindle was constructed in this paper. The influences of spindle motor drive, angle accuracy of sampling, artifact eccentricity, spindle axial motion error on the rotation accuracy were systematically studied. From the theoretical analysis and experimental results, the following conclusion remarks are obtained:

1) The cogging torque of motor can be reduced by increasing of pole pairs, so the rotation error of aerostatic spindle decreased from $0.1752 \mu \mathrm{m}$ to $0.0589 \mu \mathrm{m}$ with the growth of motor pole pairs from 10 to 64 . However, compared with the measuring results of free rotation without motor drive, the effect of motor drive on the running accuracy cannot be ignored; 
2) An angle correction algorithm was proposed to alleviate the effect of angle deviation on the measuring accuracy. With the increase of cycle number $M$ from 1 to 10, the maximum angle deviation was reduced from $0.298^{\circ}$ to $0.05^{\circ}$. And the calculation results of rotation error decreased from $61.45 \mathrm{~nm}$ to $48.71 \mathrm{~nm}$. The measuring uncertainty can be reduced by increasing the number of $M$.

3) The method of harmonic analysis was applied to investigate the effect of artifact eccentricity on the measurement results. Compared with the original measuring data, the data with the eliminated first harmonic was more close to the original measuring data at the artifact eccentricity of $0.1 \mu \mathrm{m}$, which means the accuracy of measuring results can be improved by eliminating the first harmonic. And the effect of axial motion on measuring result can be ignored by reducing the spindle axial motion to $0.2 \mu \mathrm{m}$.

\section{Acknowledgements}

The authors would like to express their sincere thanks for the support from National Natural Science Foundation of China (Grant No. U1530130), National Key R\&D Program of China (Project No. 2016YFF0102003-2), Science and Technology Support Program of Sichuan Province (Grant No. 2016RZ0047, 2016GZ0192), and Science Challenging Program of CAEP (Grant No. JCKY2016212A506-0106). 


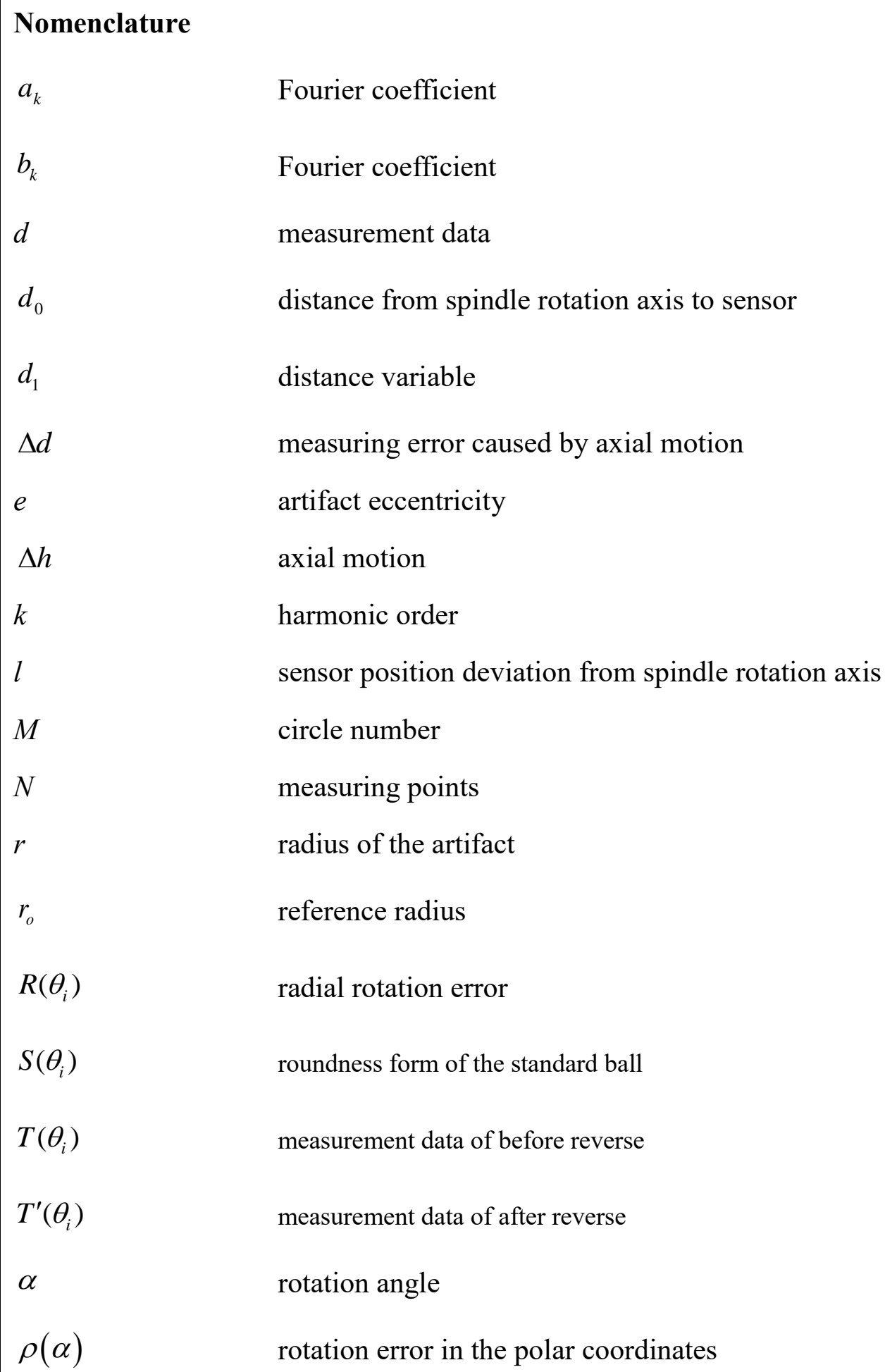




\section{References}

[1] Gao S Y, Cheng K, Ding H. CFD based investigation on influence of orifice chamber shapes for the design of aerostatic thrust bearings at ultra-high speed spindles. Tribology International. 2015; 92: 211-21.

[2] Cui H, Wang Y, Yue X, Huang M, Wang W, Jiang Z. Numerical analysis and experimental investigation into the effects of manufacturing errors on the running accuracy of the aerostatic porous spindle. Tribology International. 2018; 118: 20-36.

[3] Gao S Y, Cheng K, Ding H. Computational design and analysis of aerostatic journal bearings with application to ultra-high speed spindles. Proceedings of the IMechE, Part C: Journal of Mechanical Engineering Science. 2017; 231: 1205-1220.

[4] Cui H, Wang Y, Yue X, Huang M, Wang W. Effects of manufacturing errors on the static characteristics of aerostatic journal bearings with porous restrictor. Tribology International. 2017; 115: 246-260.

[5] Cui H, Wang Y, Yang H, Zhou L, Li H, Wang W, Zhao C. Numerical analysis and experimental research on the angular stiffness of aerostatic bearings. Tribology International. 2018; 120: 166-178.

[6] Cappa S, Reynaerts D, Al-Bender F. Reducing the Radial Error Motion of an Aerostatic Journal Bearing to a Nanometre Level: Theoretical Modelling. Tribology Letters. 2014;53:27-41.

[7] Gao W, Kiyono S, Satoh E, Sata T. Precision measurement of multi-degree-offreedom spindle errors using two-dimensional slope sensors. CIRP AnnalsManufacturing Technology. 2002;51:447-50.

[8] Evans C J, Hocken R J, Estler W T. Self-calibration: reversal, redundancy, error separation, and 'absolute testing'. CIRP Annals-Manufacturing Technology. $1996 ; 45: 617-34$.

[9] Linxiang C. The measuring accuracy of the multistep method in the error separation technique. Journal of Physics E: Scientific Instruments. 1989;22:903.

[10] Buajarern J, Somthong T, Ali S, Tonmeuanwai A. Effect of step number on roundness determination using multi-step method. International Journal of Precision Engineering and Manufacturing. 2013;14:2047-50. 
[11] Haitjema H. Revisiting the multi-step method: Enhanced error separation and reduced amount of measurements. CIRP Annals-Manufacturing Technology. 2015;64:491-4.

[12] Zhang G, Zhang Y, Yang S, Li Z. A multipoint method for spindle error motion measurement. CIRP Annals-Manufacturing Technology. 1997;46:441-5.

[13] Zhang G, Wang R. Four-point method of roundness and spindle error measurements. CIRP Annals-Manufacturing Technology. 1993;42:593-6.

[14] Okuyama E, Nosaka N, Aoki J. Radial motion measurement of a high-revolution spindle motor. Measurement. 2007;40:64-74.

[15] Gao W, Kiyono S, Satoh E, Sata T. Precision measurement of multi-degree-offreedom spindle errors using two-dimensional slope sensors. CIRP AnnalsManufacturing Technology. 2002;51:447-50.

[16] Gao W, Kiyono S. On-machine roundness measurement of cylindrical workpieces by the combined three-point method. Measurement. 1997;21:147-56.

[17] Gao W, Kiyono S, Nomura T. A new multiprobe method of roundness measurements. Precision Engineering. 1996;19:37-45.

[18] Tu J F, Bossmanns B, Hung S C. Modeling and error analysis for assessing spindle radial error motions. Precision engineering. 1997;21:90-101.

[19] Cappa S, Reynaerts D, Al-Bender F. A sub-nanometre spindle error motion separation technique. Precision Engineering. 2014;38:458-71.

[20] Shi S, Lin J, Wang X, Zhao M. A hybrid three-probe method for measuring the roundness error and the spindle error. Precision Engineering. 2016;45:403-13.

[21] Donaldson R R. Simple method for separating spindle error from test ball roundness error. California Univ., Livermore. Lawrence Livermore Lab., 1972.

[22] Grejda R, Marsh E, Vallance R. Techniques for calibrating spindles with nanometer error motion. Precision engineering. 2005;29:113-23.

[23] Marsh E R, Arneson D A, Martin D L. A comparison of reversal and multiprobe error separation. Precision Engineering. 2010;34:85-91.

[24] Marsh E, Couey J, Vallance R. Nanometer-level comparison of three spindle error motion separation techniques. ASME: Journal of Manufacturing Science and 
Engineering. 2006; 128: 180-7. 